Meta

Journal des traducteurs

Translators' Journal

\title{
Textes et termes
}

\section{Rostislav Kocourek}

Volume 36, numéro 1, mars 1991

La terminologie dans le monde : orientations et recherches

URI : https://id.erudit.org/iderudit/003330ar

Aller au sommaire du numéro

Éditeur(s)

Les Presses de l'Université de Montréal

ISSN

0026-0452 (imprimé)

Découvrir la revue

Citer cet article

Kocourek, R. (1991). Textes et termes. Meta, 36(1), 71-76.

Ce document est protégé par la loi sur le droit d'auteur. L'utilisation des services d'Érudit (y compris la reproduction) est assujettie à sa politique d'utilisation que vous pouvez consulter en ligne.

https://apropos.erudit.org/fr/usagers/politique-dutilisation/
Cet article est diffusé et préservé par Érudit.

Érudit est un consortium interuniversitaire sans but lucratif composé de l’Université de Montréal, l'Université Laval et l'Université du Québec à Montréal. Il a pour mission la promotion et la valorisation de la recherche. https://www.erudit.org/fr/ 


\section{TEXTES ET TERMES}

ROSTISLAV KOCOUREK

Université Dalhousie, Halifax, Canada

La notion de texte a marqué les années 80 de la recherche terminologique et technolinguistique. La dimension textuelle, qui a d'ailleurs toujours été respectée dans la méthodologie terminologique canado-québécoise, a enrichi la réflexion terminologique.

Le présent article traite du rapport entre les textes et les termes. Sa perspective est linguistique. Elle est donc plus étroite que l'idéal d'un domaine pluridisciplinaire terminologique (Wüster 1981), ou textologique (par exemple Pêcheux 1984).

La perspective est lexico-textuelle. Etant lexicale (terminologique), elle est plus large que l'approche uniquement textuelle plutôt alexicale (par exemple de Beaugrande et Dressler 1986). Étant textuelle, elle est moins étroite que la conception terminologique non linguistique (cf. Felber 1986: 110-111; voir le point de vue opposé de Sager 1988).

Pour plus de détails sur notre approche de la langue savante, voir nos travaux récents $(1990 \mathrm{a}, 1990 \mathrm{~b})$.

\section{TROIS FACES DU TEXTE}

L'intérêt humain principal de la linguistique, c'est qu'elle étudie le système langagier qui sous-tend des textes et qui permet d'en créer: textes parlés et écrits de la langue, résultats saisissables des activités de l'esprit. Sans textes, il n'y a pas de langue, il n'y a pas, non plus, de théorie satisfaisante de la langue.

En tant qu'une suite de phrases, liées entre elles par des connecteurs et par des élẻments sémantiques et formels communs, le texte est un phénomène complexe, dont nous observerons trois aspects, dans le domaine de la langue savante.

Le texte représente, d'abord, l'emploi des ressources de la langue sur l'axe séquentiel (syntagmatique). Il comprend des occurrences concrètes qui, ensemble, caractérisent ces ressources dans le système. Le texte-séquence est ainsi complémentaire, et opposé au système des ressources de la langue. (On lira l'article d'André Clas (1988) et l'on nous pardonnera les binominaux juxtaposés ad hoc du type texte-séquence.)

En tant que texte-séquence, le texte constitue, deuxièmement, une source de données pour l'observation de tous les plans de la langue. En tant que source de données, le texte complète la mémoire d'un analyste qui base ses observations sur sa propre compétence linguistique.

Le texte, c'est, enfin, un plan supraphrastique de l'analyse linguistique, dont la cohésion grammaticale et la cohérence sémantique sont fondées sur les plans inférieurs, mais qui les dépasse du fait qu'il possède un réseau complexe de rapports sémantiques et formels interphrastiques. Le texte-plan s'oppose ainsi à d'autres plans de la langue, surtout aux plans lexical et grammatical.

Les textes savants saisissent et expriment le contenu savant, dont les unités sémantiques dominantes sont les termes. Le terme est une unité lexicale - ou son acception - définie dans les textes savants, où apparaissent ses occurrences intégrées dans le tissu du texte. 
Quel est le rapport du terme aux trois faces du texte savant? Voici quelques remarques sur cette question, qui est essentielle pour les fondements théoriques de la linguistique de la langue savante et des études terminologiques.

\section{LE TERME ET LE TEXTE COMME SÉQUENCE D'OCCURRENCES}

Étant une unité lexicale ou son acception, le terme appartient au système de la langue, et il entre dans des rapports systémiques (paradigmatiques) divers. La dichotomie système-séquence constitue un dilemme profond de la théorie linguistique, où régnait, pendant des années, le réductivisme antitextuel saussurien et post-saussurien: seul le système de la langue était considéré comme digne d'analyse.

Nous avons noté à plusieurs reprises que le système des ressources est pour nous inséparable de la séquence textuelle que sous-tendent ces ressources (Kocourek 1990a: ch. 1.2.1). Dans cette optique épistémologique, le texte et le système sont complémentaires. Ils constituent, ensemble, la langue. Les termes ne sont pas seulement des éléments du système, mais aussi des occurrences dans les textes savants. La complémentarité de l'approche systémique et de l'approche textuelle nous semble essentielle.

\section{LE TERME ET LE TEXTE COMME SOURCE DE DONNÉES}

Dès le début des études terminologiques modernes, on témoigne une confrontation toujours renouvelée entre - d'une part - l'étude des termes basée sur l'analyse des notions et de leur système, et - d'autre part - l'étude des termes fondée sur l'observation et l'analyse de leurs occurrences dans les textes.

Nous avons proposé cette dernière approche en 1963, dans notre Habilitationsschrift manuscrite. La première de ses trois thèses était formulée ainsi (page 11, notre traduction):

\section{TRAVAIL AVEC LES SOURCES PRIMAIRES :}

Étudier les termes non seulement comme des unités de terminologie, lexicalement, mais aussi - ou, plutôt, d'abord - dans le contexte de la communication spécialisée originale; dans la construction d'une théorie, commencer par le matériau authentique, vérifier la théorie obtenue dans le matériau et l'appliquer de nouveau au matériau.

On voit que les sources primaires, la communication spécialisée originale et le matériau authentique, c'est-à-dire les textes, jouaient déjà un rôle primordial. (Voir aussi l'article de Karel Hausenblas 1963.)

Nous allons mentionner quelques problèmes terminologiques dont l'analyse est facilitée par une approche textuelle.

C'est d'abord dans les textes que se manifeste la capacité métalinguistique définitoire et redéfinitoire. Ceci permet une délimitation sémantique nouvelle du terme. La capacité langagière complémentaire, c'est-à-dire la capacité dénominatrice lexicocréatrice, permet la création de nouveaux signifiants, désignant de nouvelles notions. Une étude textuelle ponctuelle - relative d'une part aux définitions d'un signifiant donné, et d'autre part aux divers signifiants qui nomment un signifié donné - découvre une variété surprenante de faits d'ordre définitoire et dénominateur (cf. Kocourek 1989). Si l'observation des données textuelles se joint à la compétence de l'analyste, la base de réflexion systématisante est plus solide.

Est donc lié au couple définition-dénomination le processus de néologie et de son implantation. C'est surtout dans les textes que l'on trouve des solutions néologiques remarquables. Mais la première occurrence d'un néologisme défini dans le texte est - au moment de la création - un simple hapax dont le caractère éphémère est comparable au caractère fortuit, unique, d'un texte. Ce n'est que l'emploi répété du terme dans les textẹs 
différents qui mène, au fur et à mesure, à son implantation, à sa vraie terminologisation, à son passage du texte au système. Ce sont les textes spécialisés qui décident du statut systémique d'un néologisme.

Les textes contiennent une richesse de mots-lexèmes capables de collocation avec le terme dont il s'agit. Les collocations repérées complètent d'une manière nouvelle et utile notre connaissance du terme, et montrent souvent l'insuffisance de la position terminologique et lexicographique qui suppose que - puisque le terme est un invariant sémantique - son contexte est sans intérêt.

Le contexte du terme met en évidence des propriétés terminologiques graphiques, phoniques et grammaticales, telles que l'orthographe, la prononciation, le genre, le pluriel, le comportement dans les textes des syntagmes lexicaux du type langue de spécialité (nombre, article du nom modificateur). Émergent, ou apparaissent plus clairement, les problèmes syntactico-sémantiques, de pluralité et de généricité par exemple.

On trouve dans les textes aussi les mots formellement apparentés qui font partie de la même famille de mots (de termes), ex.: définition, définir, redéfinir, défini, définitoire, définitionnel, définissant, définissable, définissabilité, indéfini, indéfinissable, indéfinissabilité... De telles familles de termes sont dignes de description et d'analyse, elles reflètent diverses facettes et fonctions des notions nommées dans les textes.

L'observation des textes permet également de situer les termes thématiques d'une spécialité au sein du lexique spécialisé, et leurs rapports aux autres composantes de ce lexique: composante générale, composante générale d'orientation spécialisée, composante terminologique des domaines apparentés. La terminologic s'appuie dans les textes sur toutes sortes d'unités lexicales paraterminologiques qui n'appartiennent pas au système terminologique donné, mais qui sont indispensables dans la création d'un discours spécialisé.

L'observation des textes savants nous rappelle, en plus, une composante sousestimée de textes savants: les noms propres de chercheurs, d'organismes, d'instruments, etc. Le caractère thématique et sémantique des noms propres en fait une composante intéressante du lexique savant.

Il est permis de rappeler qu'un type prestigieux d'analyse linguistique est purement textuel: la recherche statistique de la langue savante. C'est à la base des textes savants que sont obtenues les données statistiques, par exemple celles de Lothar Hoffmann. (1982) et de Marie Tesitelová (et al. 1983).

Le dépouillement des textes spécialisés révèle une autre composante du discours scientifique dont l'existence est à peine effleurée dans les études terminologiques: il s'agit de la composante brachygraphique (arbitaire ou motivée) de la langue spécialisée: abréviations au sens large, chiffres, symboles, sigles épelés ou intégrés. Cette composante de la langue savante ne se conforme pas à la structuration usuelle de la langue. Elle constitue la transition de la langue savante naturelle au langage savant symbolique qui cohabite, dans le discours des sciences exactes surtout, avec la langue naturelle et qui donne le discours mixte langue-langage. Ce discours mixte est en dehors de notre réflexion, mais il sied de noter que certains termes nominaux dépassent les textes de la langue naturelle en participant, sous une forme brachygraphique, aux langages symboliques.

On peut alors s'attendre que l'étude des termes qui n'accepte pas, consciemment ou intuitivement, l'unité du système et du texte, risque d'arriver aux conclusions fragiles en ce qui touche au caractère des termes, de leurs définitions et sens, de leur stabilité dans le système, de leur maniabilité syntaxique, de leur fréquence, de leurs synonymes et parasynonymes, de leur monosémie et de leur biunivocité éventuelles. 
La recherche et l'observation des termes dans les textes combinent l'approche sémasiologique et l'approche onomasiologique, procèdent successivement et réciproquement du signifiant au signifié, et du signifié au signifiant. La seule approche onomasiologique n'est pas linguistiquement suffisante.

L'analyse textuelle des termes est un moyen fiable permettant de déterminer l'état actuel de l'usage et de présenter la description du terme (signifiant et signifié). L'usage est le résultat des activités variées des spécialistes, et il reflète le pluralisme et la concurrence des approches différentes. C'est pourquoi la description de l'usage précède ou accompagne l'effort normalisateur d'aujourd'hui.

Les textes font ressortir un autre aspect fondamental des termes: leur brièveté. L'avancement, le glissement du savoir - sa régression parfois - , se reflètent dans la terminologie. Ils sont concrétisés dans les textes, et le système terminologique s'adapte, se modifie, se refait constamment. C'est pourquoi une étude de la terminologie, plus encore que celle du vocabulaire fondamental non spécialisé, doit faire face à la dimension diachronique de la langue. L'obsolescence des terminologies mène au vieillissement rapide des descriptions terminologiques et des initiatives normalisatrices. Le dépouillement des textes est donc une tâche permanente, qui est aujourd'hui facilitée par le progrès des industries de la langue. Les résultats d'une analyse uniquement synchronique, qui a séduit tant de linguistes et de terminologues, sont de plus en plus éphémères, et ne saisissent qu'une partie des traits essentiels de la terminologie.

\section{LE TERME ET LE TEXTE COMME PLAN D'ANALYSE}

Il n'y a pas longtemps, maints linguistes supposaient que le plus haut niveau de l'analyse linguistique, c'est la phrase. Ce type de limitation était peu justifié et avait toutes sortes de conséquences négatives.

Aujourd'hui, la linguistique de texte, sa grammaire et sa sémantique - sous diverses étiquettes linguistiques, sémiotiques et énonciatives - est généralement acceptée comme un plan légitime d'analyse linguistique. La décennie précédente a témoigné un développement de l'approche textuelle de la langue savante : on étudiait le discours savant (ex.: Gallais-Hamonno 1982, Beacco 1982, Heslot 1983, Laurian réd. 1983, Jacobi 1984, et d'autres), et la linguistique textuelle de la langue savante (ex.: Kalverkämper 1983).

L'intérêt principal était concentré sur l'énonciation et sur les disciplines connexes de l'analyse de discours, mais il y avait aussi des études qui traitaient de la grammaire et de la sémantique textuelles, et des travaux analysant le côté syntagmatique du motlexème et du terme (par ex. : Pierre Lerat 1987, Heribert Picht 1987, Monique C. Cormier 1989, et plusieurs articles dans les actes du colloque de Montréal, 1988, André Clas réd. 1989).

L'accent sur le côté textuel de la langue savante était généralement caractérisé par un désintérêt du côté terminologique de la langue savante, dont l'importance était considérée comme dépassée. Cette tendance est compréhensible à l'étape du développement qui suit immédiatement la dominance presque absolue de la terminologie dans les études technolinguistiques. Mais, dans une optique équilibrée, la marginalisation de la terminologie est sans fondement (Kocourek 1990a : ch. 1.3.4, cf., Hoffmann 1988: 120).

Le terme est une unité sémantique fondamentale de la langue savante (système et texte). En plus de son importance au plan lexico-sémantique, le terme est l'instrument essentiel de la cohérence des textes savants, le porteur des sèmes thématiques et du contenu (cf. Drozd 1987). Il représente les nœuds du réseau isotopique, reflète le niveau raisonné d'intellectualisation et le degré circonstancié de particularisation du texte. 
Le terme a donc sa place dans l'analyse du plan textuel, il n'est pas en opposition au texte. L'étude de textes spécialisés est complémentaire à l'étude de la terminologie. Dire que l'approche terminologique a été remplacée par la linguistique des textes spécialisés n'est pas exact. C'est plutôt que l'étude de la langue spécialisée a été élargie afin de comprendre le plan textuel, et qu'elle est aujourd'hui plus complexe et plus satisfaisante.

\section{CONCLUSION}

Nous préférons donc une linguistique de langue savante qui respecte les trois faces du texte - axe séquentiel, source de données, plan supraphrastique d'analyse. Il y a douze ans, nous avons plaidé, au cours du congrès d'AILA à Montréal, pour l'approche textuelle, comme une des exigences d'analyse linguistique applicable (Kocourek 1981: 222). Ce qui était alors une prospective revêt aujourd'hui une forme plus concrète.

Outre sa valeur théorique et méthodologique, l'approche lexico-textuelle est plus pertinente dans les domaines connexes de la technolinguistique: la traduction spécialisée, la didactologie de la langue de spécialité, la lexicographie spécialisée, l'étude terminologique normalisatrice, et l'aménagement linguistique. Du point de vue théorique, notre approche réconcilie les positions terminologique et textologique en présence.

\section{BIBLIOGRAPHIE}

BEACCO, Jean-Claude (1982): Vers une description linguistique des textes historiographiques, Thèse de $3 \mathrm{e}$ cycle, Besançon, Université de Franche-Comté, Multigr.

BEAUGRANDE, Robert de, et Wolfgang DRESSLER (1986): Introduction to Text Linguistics, London, Longman.

CLAS, André (1988): «Une matrice terminogénétique en plein essor: les binominaux juxtaposés», Reiner Arntz (réd.), Textlinguistik und Fachsprache, Hildesheim, Georg Olms, pp. 215-227.

CLAS, André (réd.) (1989): «Actes du Colloque: Les terminologies spécialisées: Approches quantitative et lexico-sémantique», Meta, 34-3, pp. 335-478.

CORMIER, Monique C. (1989): «La terminologie: du terme au texte», Caroline de Schaetzen (réd.), Terminologie diachronique, Actes du colloque organisé en mars 1988 par le Centre de terminologie de Bruxelles, Bruxelles/Paris, Ministère de la communauté francophone de Belgique/CILF, pp. 212-218.

DROZD, Lubomír (1987): «Das Begriffssystem der Fachsprache als textformierende Konstituente», Manfred Sprissler (red.), Standpunkte der Fachsprachenforschung, Tübingen, Gunter Narr, pp. 107-110.

FELBER, Helmut (1986): «Einige Grundfragen der Terminologiewissenschaft aus der Sicht der allgemeinen Terminologielehre», Fachsprache (Vienne), 8-3/4, pp. 110-123.

FELBER, Helmut (1986) et Gerhard BUDIN (1989): Terminologie in Theorie und Praxis, Tübingen, Gunter Narr.

GALlAIS-HAMONNO, Janine (1982): Langage, langue et discours économique, Metz, Centre d'analyse syntaxique de l'Université de Metz.

HAUSENBLAS, Karel (1963): «Termíny a odborny text [Les termes et le texte spécialisé]», CSTC (Bratislava), 2-1, pp. 7-15.

HESLOT, Jeanne (1983): «Récit et commentaire dans un article scientifique», DRLAV (Paris), 29, pp. 133-154.

HOFFMANN, Lothar (1982): Fachwortschatz Physik, Berlin, Akademie-Verlag.

HOFFMANN, Lothar (1988): Vom Fachwort zum Fachtext, Tübingen, Gunter Narr.

JACOBI, Daniel (1984): «Du discours scientifique, de sa reformulation et de quelques usages sociaux de la science», Langue française, Paris, 64, pp. 38-51.

KALVERKÄMPER, Hartwig (1983): «Textuelle Fachsprachen-Linguistik als Aufgabe», LiLi, (Frankfurt/M), 13-51/52, pp. 124-166.

KOCOUREK, Rostislav (1981): «Prerequisites for an Applicable Linguistic Theory of Terminology», Actes du $5^{e}$ congrès de l'Association internationale de linguistique appliquée (Montréal, août 1978), Québec, Les Presses de l'Université Laval, pp. 216-228.

KOCOUREK, Rostislav (1989): "Définition, sémantique lexicale et theorie linguistique», ALFA (Halifax), 2, pp. 27-50.

KOCOUREK, Rostislav (1990a): La langue française de la technique et de la science. Vers une linguistique de la langue savante, $2^{\mathrm{e}}$ édition augmentée, refondue et mise à jour, avec une nouvelle bibliographie, Wiesbaden, Oscar Brandstetter. 
KOCOUREK, Rostislav (1990b) : «La linguistique et la langue savante», ALFA, (Halifax), 3 (à paraître).

LAURIAN, Anne-Marie (réd.) (1983): «Les discours scientifiques», Études de linguistique appliquée (Paris), 51.

LERAT, Pierre (1987): «L'acceptabilité des mots», Études de linguistique appliquée (Paris), 67, pp. 7-34.

PECHEUX, Michel (1984): «Sur les contextes épistémologiques de l'analyse de discours», Mots (Saint-Cloud), 9, pp. 7-17.

PICHT, Heriberı (1987): «Terms and their LSP Environment», Meta, 32-2, pp. 149-155.

SAGER, Juan C. (1988): «The Status of Terminology as an Independent Discipline», Parallèles (Genève), 10, pp. 21-23.

TESITELOVA, Marie (réd.) (1983): [Caractéristiques quantitatives du tchèque contemporain de spécialité (dans le cadre du style pragmatique)], Prague, Academia.

WÜSTER, Eugen (1981): «L'étude scientifique générale de la terminologie, zone frontalière entre la linguistique, la logique, l'ontologie, l'informatique et les sciences des choses», V.I. Siforov (rêd.), Fondements théoriques de la terminologie, Québec, Université Laval - GIRSTERM, pp. 55-114. 